\title{
Exploration of spatial structures made from reused elements and the design of optimal kits-of-parts
}

\author{
Jan Brütting \& Corentin Fivet \\ Structural Xploration Lab, Swiss Federal Institute of Technology (EPFL), Fribourg, Switzerland, \\ jan.bruetting@epfl.ch \\ Gennaro Senatore \\ Applied Computing and Mechanics Laboratory, Swiss Federal Institute of Technology (EPFL), Lausanne, \\ Switzerland
}

\begin{abstract}
Reuse reduces raw material use, waste generation and energy consumption caused by building construction. A substantial share of these impacts is contributed by load-bearing systems because of their mass- and energy-intensive production process. Therefore, reusing structural components over multiple service lives has the potential to improve the sustainability of building structures. However, reusing structural elements entails reversing the conventional structural design process: the mechanical and geometric properties of available elements predetermine the geometry and topology of a structure. This paper presents structural optimization techniques: 1) for the design of multiple spatial structures from one stock of elements, and 2) for the synthesis of an optimal stock or kit-of-parts whose elements can be used in multiple structures. The objective of case 1) is to avoid the cutting of stock elements, i.e. to reduce waste. In case 2), the objective is to reuse stock elements in as many structures as possible. In both cases, the assignment of stock elements to the structure is obtained via combinatorial optimization. In addition, geometry optimization is employed to best-fit the structure geometry to the lengths of assigned stock elements. The potential of the proposed methods for large-scale applications is demonstrated via case studies of three spatial structures of complex layout: a dome, two three-chord trusses and a tower.
\end{abstract}

\section{INTRODUCTION}

Recent trends in research as well as in architectural and structural design practice have identified the potential of reuse to lower the environmental footprint of the built environment (Gorgolewski, 2017). Reuse aims at avoiding raw material use for new production and at reducing the energy spent for transformations as well as at reducing waste by keeping products for multiple servicelives as close as possible to their original form. In construction, the reuse of structural components has a substantial effect, because load-bearing systems contribute the largest share to the embodied environmental impacts of building structures (Kaethner \& Burridge, 2012). For instance, reusing reclaimed steel members from disassembled buildings avoids consuming energy for re-melting and new production (Allwood and Cullen, 2012). An alternative approach to reusing reclaimed components is the synthesis of a stock of elements or kit-of-parts whose components can be reused in multiple configurations, e.g. in the case of modular or temporary structures.

As pointed out by Gorgolewski (2008), reuse entails a particular challenge: "For structural design the size and length of the available members will then determine the spans and spacing possible in the new structure [...]" (p.180). Different to conventional design, this reversed process is not supported by design guidelines or tools. The approach to facilitate the design of structures from reused elements, followed in this paper, is the application of structural optimization, which traditionally seeks best performing systems for a given set of boundary conditions and loads.

Section 2 presents two optimization methods for reticular structures subject to an element stock. The first method uses elements from a stock individually (section 2.1) and has been previously 
presented in Brütting et al. (2018). Section 2.2 extends the method to the scenario of cutting multiple members from individual stock elements. Such approach has been shown by Bukauskas et al. (2017) for the design of trusses made from timber logs by employing heuristics. Instead, this paper employs deterministic combinatorial optimization to obtain globally optimal solutions.

Section 3 introduces an optimization formulation for obtaining an optimal kit-of-parts that can be used to build multiple structures. In this method, the length and number of stock elements are the design variables of the optimization. This problem has so far received little attention. Nadir et al. (2004) have studied the reconfiguration of bars within a planar truss, in order to react to changing load actions. Tugilimana et al. (2017) considered sizing optimization, spatial reconfiguration and reuse of unit-cells in modular bridges of different spans. Basso et al. (2009) optimized the geometry of a free-form grid structure, where only a defined number of dissimilar element lengths was allowed. In contrast, the work of this paper aims at optimizing a kit-of-parts for multiple spatial structures with the objective is to maximize the number of reuse cycles per element.

\section{STRUCTURAL OPTIMIZATION WITH STOCK CONSTRAINTS}

In the following, the term member is used for a position or bar in a reticulated structure and member length is the distance between nodes at this position. The term element is used for the individual component of a stock. Figure 1(a) shows a cantilever truss with $m=5$ pin-jointed members. Two scenarios for reusing elements are considered: (A) the 1-to-1 assignment of elements to positions in the truss, and (B) a bin-packing approach, where multiple members can be cut from individual elements. Figure 1(b) relates to scenario (A) and shows Stock A with $s=7$ elements. Elements of identical properties (cross sections and lengths) are collated in $g=3$ groups. Figure 1(c) relates to scenario (B) and shows Stock B with $s=5$ elements. The color mapping between structure and stocks represents the optimal assignment and packing. Some elements have to be shortened to fit the structure geometry. In the following, such cut-off is referred to as waste.
(a) System
$m=5$ bars
$n=4$ nodes
$d=8-3=5$ unsupported dof ${ }^{\star} \mathrm{s}$

(b) Stock A - Assignment

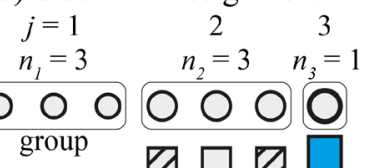

(c) Stock B - Bin-packing
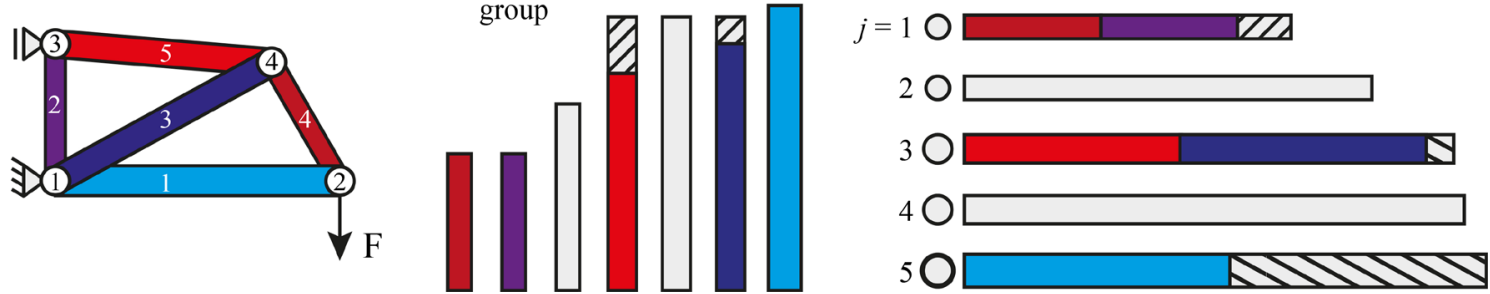

Figure 1. (a) Cantilever truss, (b) stock A and assignment, (c) stock B and bin-packing configuration

\subsection{Assignment problem}

This section presents a structural optimization formulation (A) for the assignment of stock elements to a truss. For details on the formulation the reader is referred to Brütting et al. (2018).

In general, the use of one element from stock group $j$ at position $i$ in the structure, is represented by an entry $t_{i, j}=1$ in the binary assignment matrix $\boldsymbol{T} \in\{0,1\}^{m \times g}$. The objective of the optimization is to avoid waste $W$ (Eq. 1) via minimizing the length distance between members and stock elements, i.e. between $\overline{\boldsymbol{l}}$ and $\boldsymbol{l}$. To obtain a measure in terms of mass, the length difference is weighted by the assigned element cross section areas $\boldsymbol{a}$ and material density $\rho$. The operator $\circ$ in Eq. (1) indicates an element-wise multiplication of vector entries.

A simultaneous analysis and design approach is followed where the structural analysis is part of the optimization in the form of constraints (Haftka, 1985). For each load case $k$, equilibrium of forces and stress constraints are taken into account via Eqs. (2) and (3). Eq. (4) guarantees the assignment of exactly one stock element at each position, i.e. the topology of the system is invariant. Eq. (5) constrains the element assignment to $n_{j}$, which is the number of available elements 
per group. Eq. (6) imposes that assigned elements need to be longer or equal to the nodal distances (member lengths $\overline{\boldsymbol{l}}$ ) of the corresponding truss positions.

(A)

$$
\begin{aligned}
& \min _{\boldsymbol{T}, \boldsymbol{p}} W=\rho \underline{\mathbf{1}}^{T} \boldsymbol{T}(\boldsymbol{a} \circ \boldsymbol{l})-\rho \overline{\boldsymbol{l}}^{T} \boldsymbol{T a} \\
& \text { s.t.: } \boldsymbol{B} \boldsymbol{p}^{(k)}=\boldsymbol{f}^{(k)} \forall k \\
&-\sigma \boldsymbol{T} \boldsymbol{a} \leq \boldsymbol{p}^{(k)} \leq+\sigma \boldsymbol{T} \boldsymbol{a} \forall k \\
& \sum_{j=1}^{g} t_{i, j}=1 \forall i=1 \ldots m \\
& \sum_{i=1}^{m} t_{i, j} \leq n_{j} \forall j=1 \ldots g \\
& \overline{\boldsymbol{l}} \leq \boldsymbol{T} \boldsymbol{l}
\end{aligned}
$$

Stock vectors: $\boldsymbol{a}, \boldsymbol{l} \in \mathbb{R}^{g} ;$ member lengths $\overline{\boldsymbol{l}} \in \mathbb{R}^{m} ;$ equilibrium matrix: $\boldsymbol{B} \in \mathbb{R}^{d \times m} ;$ member forces $\boldsymbol{p} \in \mathbb{R}^{m}$; ext. forces: $\boldsymbol{f} \in \mathbb{R}^{d}$; material strength: $\sigma$; vector of all ones: $\underline{\mathbf{1}} \in\{1\}^{m}$

The formulations of this paper omit geometric compatibility and nodal displacements (plastic design). Therefore, obtained structures might for instance violate deflection constraints. Yet, plastic design and invariant topology reduce the computational complexity allowing this method to be scalable to large-scale spatial systems. If deflection constraints would be governing a design, the elastic formulations given in Brütting et al. (2018) should be employed in a post process.

Problem (A) is a Mixed-Integer Linear Programming (MILP) problem and can be solved to global optimality, for instance via branch-and-bound techniques (Nemhauser and Wolsey, 1988).

\subsection{Bin-packing problem}

The bin-packing formulation (B) is directly developed from (A). Cutting of multiple members from one element requires treating every stock element individually $(j=1 \ldots s)$ instead of in groups. This entails expanding all stock vectors from size $g$ to size $s$ and the size of $\boldsymbol{T}$ becomes $m \times s$. In addition, binary variables $\boldsymbol{y} \in\{0,1\}^{s}$ are included to identify whether one or more members are cut from an element $\left(y_{j}=1\right)$ or conversely whether an element remains unused $\left(y_{j}=0\right)$. The inclusion of $\boldsymbol{y}$ is required because in (B) multiple truss member might be paired with one stock element, which would result in double counting when using Eq. (1) instead of Eq. (7). Further, Eq. (8) replaces Eq. (6) since for bin-packing the sum of the member lengths must be smaller or equal to the length of the stock element to which the members are assigned.

$$
\begin{aligned}
& \qquad \min _{\boldsymbol{T}, \boldsymbol{y}, \boldsymbol{p}} W=\rho \boldsymbol{y}^{T}(\boldsymbol{a} \circ \boldsymbol{l})-\rho \overline{\boldsymbol{l}}^{T} \boldsymbol{T} \boldsymbol{a} \\
& \text { s.t.: Eqs. (2), (3) and (4) } \\
& \boldsymbol{T}^{T} \overline{\boldsymbol{l}} \leq \boldsymbol{y} \circ \boldsymbol{l}
\end{aligned}
$$

(B) is a standard MILP problem, yet the expansion of $\boldsymbol{T}$ and the addition of binary variables $\boldsymbol{y}$ increases the problem size with respect to (A), which might result in longer computations.

\subsection{Geometry optimization}

After solving problems (A) or (B), the lengths of the selected elements may not match exactly the member lengths of the structure. For the globally optimal assignment or bin-packing solutions, geometry optimization is then carried out to minimize cut-off waste (Eqs. (1) or (7)) by varying the structure node locations (coordinates). To maintain geometric features of an initial design intention, it is possible to restrict node locations to given domains. Solving problems (A) or (B) and employing geometry optimization is sequentially executed over multiple iterations until convergence is reached, i.e. until no further waste reductions are achieved (Brütting et al., 2018). 


\section{STOCK OPTIMIZATION}

This section introduces a formulation (S) to optimize the configuration of a stock or kit-of-parts such that its elements can be reused in various structures. Problem (S) can be though as the "inverse" of (A) and includes the stock element lengths $\boldsymbol{l}$ and the number of elements per group $\boldsymbol{n}$ as design variables. This means, for a predefined set of $g$ groups with cross-section areas $\boldsymbol{a}$, that the optimal number of elements per group and their length is determined.

The different structural systems intended to be built from the kit-of-parts are collected in the set $R$. Problem (S) directly builds on formulation (A) since individual elements should be used multiple times and without cutting, i.e. the bin-packing scenario is not applicable here.

$$
\begin{aligned}
& \min _{\boldsymbol{T}, \boldsymbol{l}, \boldsymbol{n}, \boldsymbol{p}} w_{1} N_{t o t}+w_{2} W_{t o t}=w_{1} \sum_{j=1}^{s} n_{j}+w_{2} \sum_{r=1}^{R} W^{r} \\
& \text { s.t.: Eqs. (2), (3) and (4) } \forall r=1 \ldots R \\
& \sum_{i=1}^{m} t_{i, j}^{r} \leq n_{j} \forall j=1 \ldots g ; r=1 \ldots R \\
& \boldsymbol{T}^{r} \boldsymbol{l}-\Delta \leq \overline{\boldsymbol{l}}^{r} \leq \boldsymbol{T}^{r} \boldsymbol{l} \forall r=1 \ldots R
\end{aligned}
$$

Optimizing the stock configuration is carried out by combining multiple objectives (Eq. 9) via weighting factors $\left(w_{1}\right.$ and $\left.w_{2}\right)$. The first objective is the minimization of the total number of stock members $N_{t o t}$. Minimizing $N_{t o t}$ entails that elements are reused as often as possible in multiple structures. At the same time, element cutting has to be avoided, i.e. cut-off waste $W^{r}$ must be minimized for every structural system and assignment (see Eq. (1) for the computation of $W^{r}$ ).

Eq. (10) relates the assigned elements per system with the total number $n_{j}$ of elements per stock group. Eq. (11) constrains the assignment and length variables: member lengths $\overline{\boldsymbol{l}}^{r}$ must be within a defined range $\Delta$ to the stock element lengths $\boldsymbol{l}$ (design variables). Here, $\Delta$ represents a length distance or tolerance that, for instance, could be compensated via adjustable joints.

Formulation (S) contains products of binary variables $t_{i, j}$ and continuous variables $l_{j}$. Such "bilinear" terms can be replaced via continuous auxiliary variables and linear constraints (Glover, 1984). This linearization allows expressing (S) as a MILP problem in standard form.

Similar to the methods presented in section 2, once (S) is solved to optimality, geometry optimization is carried out to match node positions and element lengths. To achieve an improved fitting between structure geometry and kit-of-parts, the stock element lengths $\boldsymbol{l}$ are also included as design variables in the geometry optimization step. Starting from a larger value, during the optimization sequence $\Delta$ is decreased at each iteration to obtain a final result where all elements are used at their full length $(\Delta=0)$ or within a defined tolerance.

\section{RESULTS}

\subsection{Three spatial structures made from one stock}

This case study considers the consecutive construction of three spatial structures from one element stock. This setting entails that if element cutting is required for one system, in the next use only the remaining element lengths are available. A possible scenario for this case study would be the building of multiple (temporary) systems while reusing elements from the same stock.

Table 1 gives metrics for an assumed stock of 200 elements with circular hollow sections of grade S235 steel. Cross sections of six different sizes are available. Per section, up to two different element lengths are considered, resulting in a stock with 10 groups in total.

Table 1. Stock characteristics - circular hollow sections.

\begin{tabular}{llllllllll|lll}
\hline Group & & & 1 & 2 & 3 & 4 & 5 & 6 & 7 & 8 & 9 & 10 \\
\hline Areas & $\boldsymbol{a}$ & {$\left[\mathrm{cm}^{2}\right]$} & \multicolumn{2}{c}{1.82} & \multicolumn{3}{c}{3.07} & \multicolumn{2}{c}{3.94} & 5.87 & 5.57 & 8.31 \\
Lengths & $\boldsymbol{l}$ & {$[\mathrm{m}]$} & 1.50 & 2.25 & 2.00 & 1.20 & 1.60 & 1.90 & 1.80 & 2.00 & 2.20 & 1.50 \\
Availability & $\boldsymbol{n}$ & {$[-]$} & 20 & 20 & 20 & 20 & 20 & 20 & 20 & 20 & 20 & 20 \\
\hline
\end{tabular}


Figure 2 shows the three spatial structures envisioned for this case study: 1) a dome, 2) two arched trusses, and 3) a tower. System dimensions, loading and support conditions are indicated in Figure 2. The design domains represent the considered geometry optimization constraints for each system. For instance, the nodes of the dome (incl. supports) must lie on a spherical surface with a radius between $4.50 \mathrm{~m}$ and $5.50 \mathrm{~m}$. The arched trusses' bottom chord nodes are constrained to a vertical plane, whereas their top chord node positions are fixed to maintain the arch shape. For each level of the tower, all nodes have to remain within horizontal rings, having a maximum radius of $2.30 \mathrm{~m}$. The tower height is restricted to $10.5 \mathrm{~m}$.
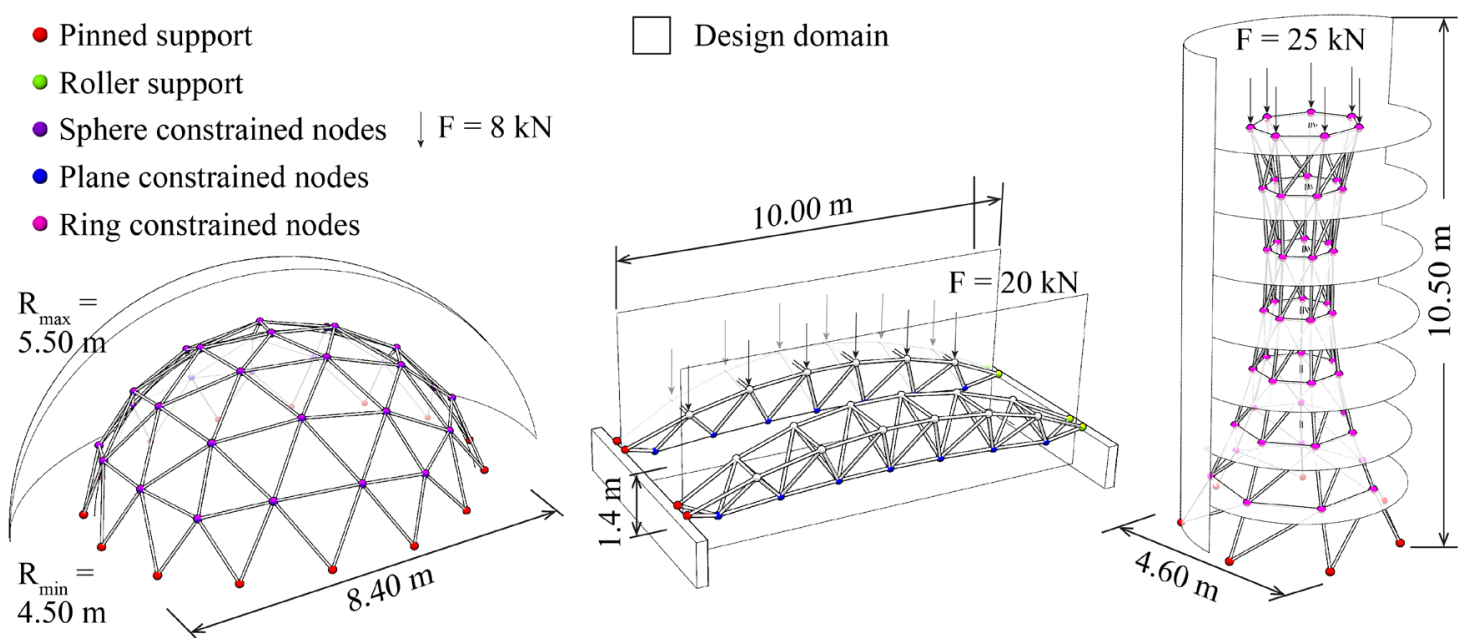

Figure 2. System layouts, support conditions, geometric constraints and assumed loads.

The systems are defined parametrically to vary e.g. the radius of the dome, the number of bays in the arched trusses or the number of tower levels. This parametric setup facilitates the easy adaption of the layouts to attain geometries where all members are shorter but closely related to the available stock element lengths. Table 2 compares metrics of the stock with those of the structures. The systems were defined such that the mean member length decreases from the dome over the arched trusses to the tower. This way, it is possible to build the systems in this consecutive order.

Table 2. Number of elements and length distribution for stock and structures.

\begin{tabular}{lllllll}
\hline Metric & Unit & Stock & Dome & Trusses & Tower & All systems \\
\hline \# members & {$[-]$} & 200 & 105 & $2 \times 54$ & 147 & 360 \\
$\min l$ & {$[\mathrm{~m}]$} & 1.20 & 1.57 & 0.91 & 0.57 & 0.57 \\
$\operatorname{mean} l$ & {$[\mathrm{~m}]$} & 1.78 & 1.76 & 1.41 & 1.27 & 1.46 \\
$\max l$ & {$[\mathrm{~m}]$} & 2.25 & 1.86 & 1.68 & 2.15 & 2.15 \\
\hline
\end{tabular}

For all three systems, assignment (A) and bin-packing optimization (B) are employed in combination with geometry optimization. Results are summarized in Table 3 . The optimal assignment and geometry of the dome requires cutting 29 elements (tolerance $1 \mathrm{~mm}$ ), which results in $10.6 \mathrm{~kg}$ of waste. Employing assignment or bin-packing converges to the identical solution because the dome members are generally long and therefore no stock elements could be partitioned via (B).

For the arched trusses, the number of off-cuts in scenarios (A) and (B) are in the same order of magnitude. However, bin-packing successfully reduces waste from $19.9 \mathrm{~kg}$ to $9.0 \mathrm{~kg}$. Employing (A) to the tower results in a large number of cuts and the most waste because many short members are present in the tower rings. Instead, applying bin-packing to the tower results in the least waste of all cases.

Table 3. Assignment and bin-packing results for all three structures.

\begin{tabular}{lllllll}
\hline Metric & Unit & Dome (A) and (B) & Trusses (A) & Trusses (B) & Tower (A) & Tower (B) \\
\hline \# off-cuts & {$[-]$} & 29 & 56 & 60 & 82 & 19 \\
max. $\Delta$ & {$[\mathrm{cm}]$} & 64.6 & 98.5 & 36.1 & 104.6 & 5.8 \\
Waste & {$[\mathrm{kg}]$} & 10.6 & 19.9 & 9.0 & 22.5 & 1.1 \\
\hline
\end{tabular}


Figure 3 indicates the consecutive use of the stock for the three structures. In particular, the cut and left over elements of dome (A) become the stock for the arched trusses (A) which then give the stock for the tower (B). Figure 3(a) shows the optimal geometry of the dome, whose nodes are on a spherical surface with a new radius of $4.66 \mathrm{~m}$. For comparison, the initial structure geometry (input) is indicated in light grey. The elements used for the dome are represented via the colored bars in the stock diagram on the right side of Figure 3(a).

Figure 3(b) indicates the structure geometry and 1-to-1 assignment for the two arched trusses. Via geometry optimization, the bottom chord nodes are shifted downwards to best-fit the assigned element lengths. However, because the top chord nodes are defined immovable, many elements require cutting. A relaxation of these geometric constraints might reduce cutting and waste.

The optimized geometry and the bin-packing solution for the tower structure are shown in Figure 3(c). In total 37 stock members are effectively divided into two pieces, resulting in almost no cut-off waste overall. Only from elements of groups 1,2 and 3 short pieces are cut off ( Table $3)$. The bin-packing method further facilitates that the final tower geometry remains close to the initial design input, for instance the total height of the tower is only merely increased.
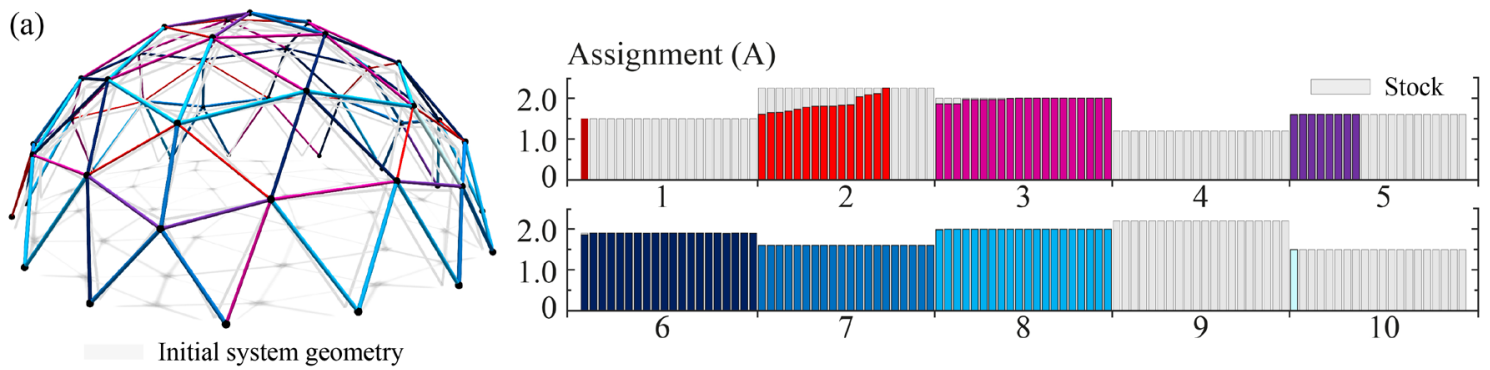

(b)
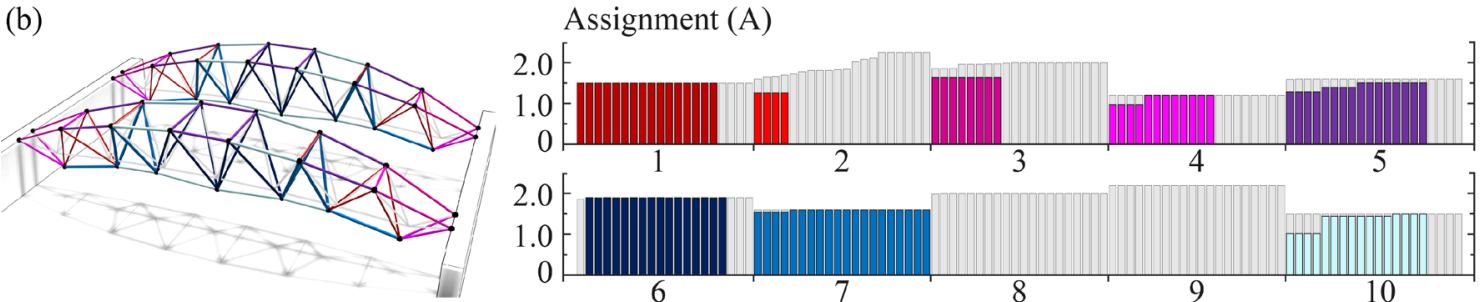

(c)
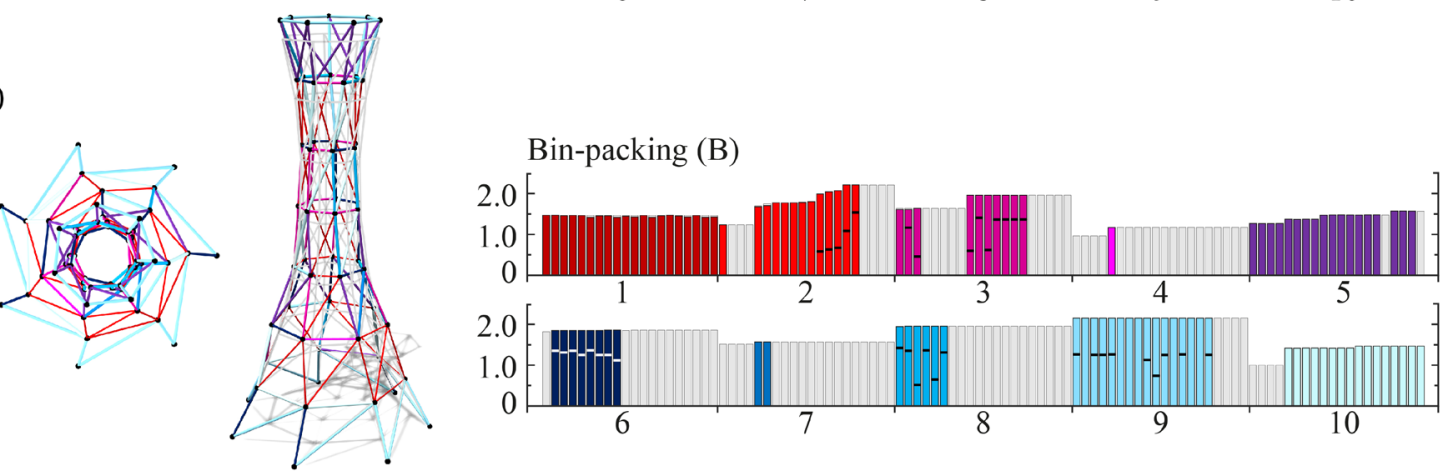

Figure 3. Optimal structure geometries and stock use: (a) dome, (b) trusses, and (c) tower.

\subsection{One stock for three systems}

This section presents the optimal synthesis of an element stock that permits building the three spatial structures of previous section 4.1 in arbitrary order and without cutting any elements. To attain such optimal kit-of-parts, the method of section 3 is employed.

In this section in total five cases are studied. Cases 1) - 4) consider the same stock composition as reported in Table 1, i.e. a stock with ten groups of six different section sizes is the input. The optimal element lengths and numbers per group are then determined by employing method (S) and geometry optimization. In addition, case 5) represents a setting with only six stock groups in order to reduce the number of different building components. Table 4 summarizes all cases and gives characteristics of the resulting stocks. A discussion of each of the five cases follows thereafter. 
Table 4. Optimization results for the element stock.

\begin{tabular}{lllllllll}
\hline Case & Groups & Constraints & $w_{1}$ & $w_{2}$ & $N_{\text {tot }}$ & $\begin{array}{l}W_{\text {tot }} \\
{[\mathrm{kg}]}\end{array}$ & \# cuts & $\begin{array}{l}\max \Delta \\
{[\mathrm{cm}]}\end{array}$ \\
\hline 1$)$ & 10 & original & 0.1 & 1 & 241 & 4.1 & 46 & 8.2 \\
$2)$ & 10 & relaxed & 1.0 & 0 & 215 & 1.1 & 14 & 2.5 \\
$3)$ & 10 & relaxed & 0.2 & 1 & 223 & 0.1 & 4 & 0.5 \\
$4)$ & 10 & relaxed & 0.1 & 1 & 231 & 0 & 0 & 0 \\
$5)$ & 6 & relaxed & 0.1 & 1 & 217 & 2.1 & 21 & 6.0 \\
\hline
\end{tabular}

Case 1) is based on the three spatial structures with their original design domains (section 4.1). For case 1) it is not possible to avoid element cutting completely, i.e. $\Delta$ cannot be reduced to zero in successive iterations (Eq. 11). In total, 46 element lengths do not match with the member lengths of the structures. Therefore, a relaxation of the geometric constraints is considered for cases 2) - 5): the dome nodes are allowed to move up to $+10 \mathrm{~cm}$ in normal direction to a spherical surface, and the arched truss top chord nodes are allowed to move $\pm 10 \mathrm{~cm}$ in all directions.

Table 4 gives metrics for cases 2) - 4) in view of different weighting factors $w_{1}$ and $w_{2}$ considered in (S). In case 2) minimization of stock members is the sole objective $\left(w_{2}=0\right)$ and the optimal stock consists of 215 elements. However, still 14 stock elements differ up to $2.5 \mathrm{~cm}$ from the structure member lengths. In case 3) where waste minimization is included into the objective function via $w_{2}$, the total number of elements increases to 223 . Yet, including waste minimization achieves a reduced length discrepancy of $0.5 \mathrm{~cm}$ for only four elements.

In case 4), the weighting factor $w_{l}$ on $N_{\text {tot }}$ is further decreased and a solution that avoids any cutting is achieved. The optimal kit-of-parts consist of 231 elements and permits building each of the three structures (compare to \#members in Table 2). In practice, when small length differences might be compensated via adjustable joints, also cases 2) and 3) could be a solution.

Figure 4(a) shows the obtained optimal structure geometries for case 4). The relaxation of the geometric constraints results in different optimal layouts than those obtained in section 4.1. In particular, the arched truss top chord nodes shift away from the arch shape and the top part of the tower turns into a more cylindrical shape to be made by elements of similar lengths.

The characteristics of the kit-of-parts for case 4) are indicated in Figure 4(b), where colored bars represent the element lengths per group and corresponding labels indicate the number of elements used per structure. Numbers in rectangles corresponds to the maximum amount of elements used per group, i.e. the quantity of elements that needs to be manufactured for the kit-ofparts. The assignment of elements is indicated via the color mapping onto the structures.

(a)

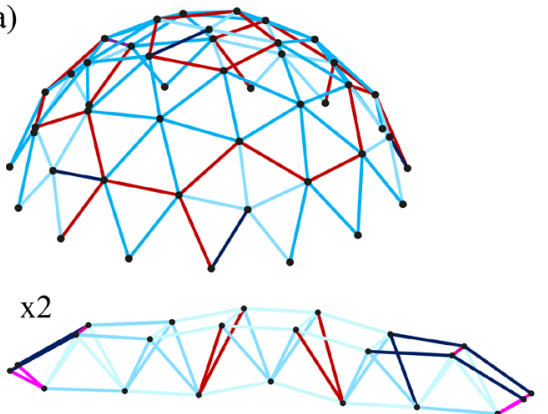

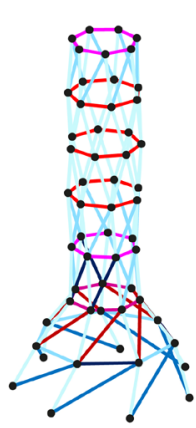

(b)

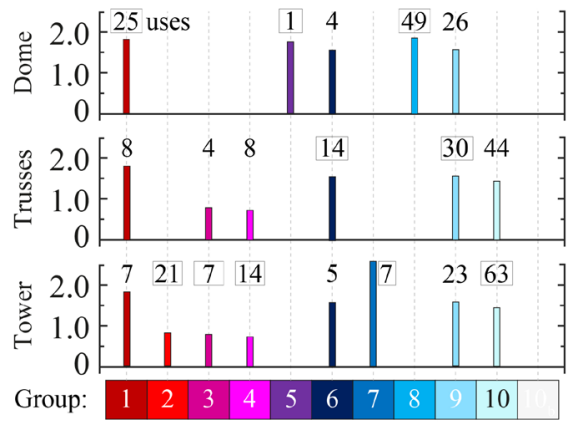

Figure 4. Optimal stock for case 4). (a) System geometries, (b) elements used per system and group.

In case 4), the dome is mainly composed of elements from groups 1, 8 and 9. In particular, all elements of group 8 are exclusively used in the dome. The arched trusses employ elements from groups 9 and 10, which are also shared with the tower. Short elements of groups 2, 3, and 4 are primarily used for the rings of the tower. The stock consist of more elements with larger cross section sizes (groups 8-10) because their higher capacity allows their reuse in more positions.

Figure 4(b) further indicates that in the kit-of-parts the elements of groups 1 and 8 as well as 5 and 9 have similar lengths. This means that it might be possible to merge these groups in order to reduce the number of different element types.

Case 5) considers a setting with only six different element types. In this case three cross section sizes, the ones of former groups 5/6,7/8, and 10 ( Table 1 ), and two lengths per cross-section are 
allowed. The optimum stock of case 5) contains 217 elements, where for 12 members a length mismatch of $6.0 \mathrm{~cm}$ to the assigned elements exists (Table 4). If such gap is admissible, the structures shown in Figure 5(a) could be built via the indicated kit-of-parts. For only six element types, no solution with exactly matching lengths could be achieved within the setup of this case study.

(a)

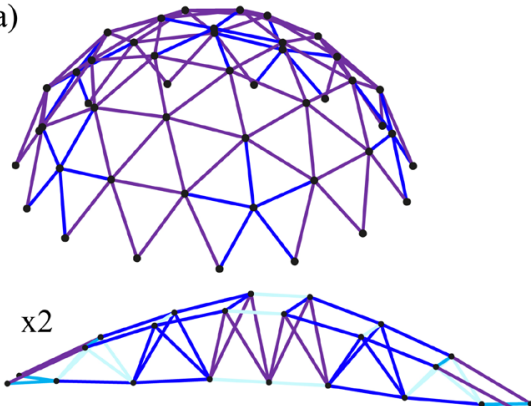

Figure 5. Optimal stock with six groups.

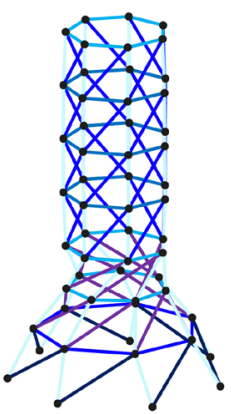

(b)

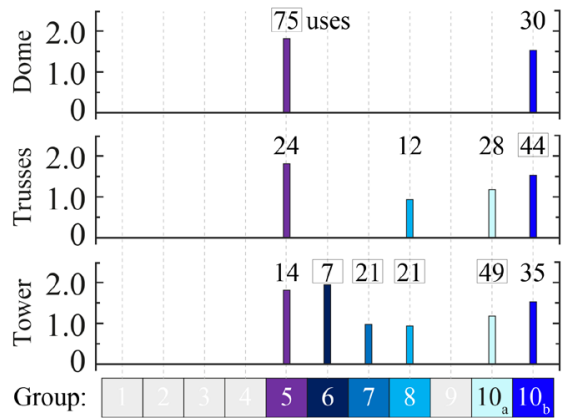

(a) System geometries, (b) elements used per system and group.

\section{CONCLUSION}

This paper presents structural optimization techniques for the design of spatial structures from reused elements. An extension of the method to the bin-packing scenario whereby multiple components can be extracted from one stock element gives improved results. Further, an effective method for synthesizing an optimal kit-of-parts whose elements can be reused in multiple structures is presented.

This paper focuses only on waste reduction, which does not explicitly minimize the structure mass. Future work could incorporate mass minimization within a multi-objective optimization. This is of particular interest for synthesizing kits-of-parts by including cross section areas as design variables.

In this paper, geometric compatibility, nodal displacements and deflection limits are omitted. This simplification allowed the application of the proposed methods to large-scale spatial structures. Future work should study the influence of this simplification on the method.

Lastly, in both cases, reuse from a given stock and synthesizing a kit-of-parts, members have to be connected under various angles at nodes with different valences. Therefore, a key challenge for the realization of this work in practice is the design and manufacturing of joints.

\section{REFERENCES}

Allwood, J.M., Cullen, J.M. 2012. Sustainable materials: with both eyes open. Cambridge: UIT Cambridge.

Basso, P., Del Grosso, A.E., Pugnale, A., Sassone, M. 2009. Computational morphogenesis in architecture: Cost optimization of free-form grid shells. J IASS 50: 143-150.

Brütting, J., Desruelle, J., Senatore, G., Fivet, C. 2018. Design of Truss Structures Through Reuse. Structures. Doi: https://doi.org/10.1016/j.istruc.2018.11.006 (in press)

Bukauskas, A., Shepherd, P., Walker, P., Sharma, B., Bregula, J. 2017. Form-Fitting strategies for diversitytolerant design. In Proceedings of the IASS Annual Symposium. Hamburg.

Glover, F. 1984. An improved MIP formulation for products of discrete and continuous variables. Journal of Information and Optimization Sciences 5: 69-71.

Gorgolewski, M. 2008. Designing with reused building components: some challenges. Building Research \& Information 36: 175-188.

Gorgolewski, M. 2017. Resource Salvation: The Architecture of Reuse. Hoboken: John Wiley \& Sons.

Haftka, R.T. 1985. Simultaneous analysis and design. AIAA journal 23: 1099-1103.

Kaethner, S. \& Burridge, J. 2012. Embodied CO2 of structural frames. The structural engineer 90: 33-40.

Nadir, W., Kim, I.Y., Hauser, D., de Weck, O. 2004. Multidisciplinary structural truss topology optimization for reconfigurability. In 10th Multidisciplinary Analysis and Optimization Conference. Albany.

Nemhauser, G.L. \& Wolsey, L.A. 1988. Integer programming and combinatorial optimization. Wiley.

Tugilimana, A., Thrall, A.P., Filomeno Coelho, R. 2017. Conceptual Design of Modular Bridges Including Layout Optimization and Component Reusability. Journal of Bridge Engineering 22(11): 04017094. 